\title{
On inverse analysis and robustness evaluation for biological structure behaviour in FE simulation - Application to the liver
}

\author{
Cécile CONTE*, Catherine MASSON, Pierre-Jean ARNOUX
}

Laboratoire de Biomécanique Appliquée, INRETS/Université de la Méditerranée, Marseille, France

Faculté de Médecine - Secteur Nord

Bd P. Dramard

13916 Marseille, France

*Corresponding author email : cecile.conte@inrets.fr

(Received XX Month Year; final version received XX Month Year)

\begin{abstract}
To prevent abdominal organs traumas, the definition of efficient safety devices should be based on a detailed knowledge of injury mechanisms and related injury criteria. In this sense, FE simulation coupled to experiment could be a valuable tool to provide a better understanding of internal organs behaviour under crash conditions.

This work proposes a methodology based on inverse analysis which combines exploration process optimisation and robustness study to obtain mechanical behaviour of the complex structure of the liver through FE simulation. The liver characterisation was build on Mooney Rivlin hyperelastic behaviour law considering whole liver structure under uniform quasi-static compression. With the global method used, the model fits experimental data. The variability induced by modelling parameters is quantified within a reasonable time.
\end{abstract}

Keywords: liver compression, FE simulation, inverse analysis, robustness analysis

\section{Introduction}

Abdominal organs and particularly the liver are highly injured during crash situations leading to severe traumas with high morbidity (Laumon 2002). To prevent this, modelling can provide efficient tools for injuries prediction once it is well calibrated. The current simulation tools suffer from the lack of data concerning dynamical loading and damage and failure identification (Haug et al. 2004).

The liver is a complex structure considered as an assembly of three tissues: parenchyma making up most of its volume, vascular and biliary structures forming a ramified network within parenchyma, and Glisson's capsule wrapping the whole organ and insuring its cohesion (Netter 1999). Hence, the direct identification of liver hyperelastic or viscoelastic mechanical behaviour becomes tricky considering this complex structure. To achieve these objectives, some works simplify the geometry by 
studying tissues samples (Chui et al. 2007, Saraf et al. 2007) or by freeing from structure effects and measuring local behaviour (Carter et al. 2001, Mazza et al. 2007). In both cases the local information obtained has to be extended at the structure level considering whole organ shape and border effects.

Another solution consists to use inverse analysis (Miller 2000, Tillier et al. 2003, Durand-Reville et al. 2004, Egorov et al. 2008) at the structure level. A numerical model of the liver governed by a finite number of parameters must then be built. Model response is compared to different target responses obtained experimentally so that parameters values can be isolated to be those for which the model best fit the experimental reality. This last solution is easier to implement than the direct characterization but is subject to uncertainties created by the induced variability of numerical phenomenon inherent in modelling.

The objective of this work is to define a methodology based on inverse and robustness analysis to obtain mechanical behaviour of the complex structure of the liver through FE simulation. The inverse analysis is applied to the identification of a hyperelastic behaviour of the whole liver under quasi-static loadings up to damage and failure. Then the robustness analysis is performed to quantify the variability induced by all modelling parameters to model response.

\section{Materials \& Methods}

\subsection{Testing conditions}

The reference test used in this work was a uniaxial quasi-static rear to front compression: the liver lied on is posterior face and was compressed between two horizontal plates (Figure 1) at a uniform velocity of $80 \mathrm{~mm} / \mathrm{min}$. The experimental test was made with a hydraulic press on a whole liver sampled on PMHS (Post Mortem Human Subject) female, 81 years old. The PMHS (obtained from the 
Marseilles University Faculty of Medicine) was treated with Winckler solution

(Winckler 1974) to ensure proper preservation of soft tissues and then held at $+3^{\circ} \mathrm{C}$ until liver removal. In order to avoid organ degradation out of the body, the test was performed few hours after the organ removal from the cadaver abdomen. The two plates between which the liver was compressed were first coated with petroleum jelly to avoid frictions with the organ surface. Compression was carried out until $60 \%$ of the initial thickness with no initial preconditioning.

The simulation test was performed using a Finite Element (FE) model built from CT-scans data (Labé et al. 2006). This model includes a volume part meshed with tetrahedral elements describing the parenchyma and a surface part describing the Glisson's capsule using a continuous 3D surface mesh. The mesh size used for these simulations is about $4 \mathrm{~mm}$. The Glisson's capsule was assumed as elastic whereas parenchyma was set hyperelastic with a Mooney-Rivlin law commonly used to describe biological materials (Miller and Chinzei 1997, Farshad et al. 1999, Tillier et al. 2003, Miller 2005, Snedeker et al. 2005, Harrison et al. 2007). The Helmholtz free energy function then follows equation (1).

$$
W=C_{10}\left(I_{1}-3\right)+C_{01}\left(I_{2}-3\right)
$$

where $I_{1}$ et $I_{2}$ are the two first invariants of the Cauchy tensor $\underline{\underline{C}}$ defined by equation (2) and $C_{10}$ et $C_{01}$ are two parameters of the law expressed in MPa..

$$
\begin{aligned}
& I_{1}=\operatorname{tr}(\underline{\underline{C}}) \\
& I_{2}=\frac{I_{1}^{2}-\operatorname{tr}\left(\underline{\underline{C}}^{2}\right)}{2}
\end{aligned}
$$

For the reference compression case, the plates set as rigid, were kept parallel one to the other during the whole simulation. As the lower plate is fixed, the upper plate velocity is $0.2 \mathrm{~m} \cdot \mathrm{s}^{-1}$. Symmetrical interfaces between liver and plates were defined and consisted in two unilateral contacts without Coulomb friction. Penalty 
algorithms detected both plates' nodes and edges penetrating a $0.3 \mathrm{~mm}$ gap between liver and plates.

\subsection{Inverse analysis}

After a convergence analysis confirming that $4 \mathrm{~mm}$ element size was effectively in the mesh convergence area of the model, the inverse analysis process followed three steps.

\subsubsection{Influent parameters determination: exploration step}

The first step of the inverse analysis was the determination of the influent parameters for the model response. For this model the potential influent parameters are the parenchyma density $d$, Poisson coefficient $v$ and the two modules of the hyperelastic law $C_{10}$ and $C_{01}$. The space of potential responses of the model for these four parameters was explored using a two levels full factorial design of experiments $2^{4}$ without repetitions (Schmidt and Launsby 1994). These levels are the extremities of our exploration space and are chosen as follows:

- Density is varying between 0.005 and $0.0015 \mathrm{~g} . \mathrm{mm}^{-3}$ around water density,

- Poisson coefficient is varying between 0.2 and 0.495 to cover a broad range from compressibility to incompressibility,

- Moduli are varying between 0.001 and $0.1 \mathrm{MPa}$ to define a range that includes values already used for biological materials in literature (Miller and Chinzei 1997, Miller 2000, Snedeker et al. 2005).

The response of a given treatment was the evaluation function $Y$ defined by

equation (3). $Y$ was the average of relative difference between the force needed to reach a relative displacement $u_{i}$ in the treatment configuration $F_{\text {treat }}\left(u_{i}\right)$ and in a reference one $F_{\text {ref }}\left(u_{i}\right)$ chosen arbitrarily with plausible values of the parameters.

$$
Y=\frac{1}{N} \sum_{i=1}^{N} \frac{\left|F_{\text {treat }}\left(u_{i}\right)-F_{\text {ref }}\left(u_{i}\right)\right|}{F_{\text {ref }}\left(u_{i}\right)}
$$

$N$ is the number of points of the response curve. The reference configuration is: $d=$ 0.001 g.mm $\mathrm{m}^{-3}, v=0,495, C_{10}=0.003 \mathrm{MPa}$ and $C_{01}=0.011 \mathrm{MPa}$. 
The aim of this step is to narrow the space of search for the set of parameters that best fit the experimental data. Influent parameters are discriminated as being the significant variables of a linear regression ( $\mathrm{p}$-value $<0.01)$. Hence, their variation ranges could be bounded comparing the responses curves with an experimental one that is representative of this solicitation speed.

\subsubsection{Model fitting with experiment: optimisation step}

From the space of candidate solutions, a NLPQL optimisation algorithm (Schittkowski 1985-1986) was used to find the set of parameters which best fit to the experimental data. The optimisation function definition was the same as for the evaluation function replacing the reference curve in equation (3) with the experimental curve of the Erreur ! Source du renvoi introuvable. and the Erreur ! Source du renvoi introuvable. :

$$
Y_{\text {opt }}=\frac{1}{N} \sum_{i=1}^{N} \frac{\left|F_{\text {treat }}\left(u_{i}\right)-F_{\text {exp }}\left(u_{i}\right)\right|}{F_{\text {exp }}\left(u_{i}\right)} \leq 0.2
$$

\subsection{Robustness evaluation}

The last step of the process was a robustness evaluation of the optimized model obtained after the inverse analysis. This evaluation was then performed on the reliability of the model parameters, of the geometrical modelling choices, and on the running simulation variables. The evaluation function introduced in equation (3) was used a new time to quantify the difference in model responses induced by parameters variations taking the response of the optimized model as reference data.

\subsubsection{Mechanical parameters}

The investigations are performed on the influent parameters isolated in the exploration step considering $\pm 10 \%$ variation around their optimal value. This variation range seems large enough to check the model stability. The space of study 
then defined was described with a minimal number of treatments using an Optimal

Latin Hypercube sampling (Sacks et al. 1989, Park 1994).

\subsubsection{Boundary conditions parameters}

By reference to experimental set up, boundary conditions parameters (candidates to dispersion) were the horizontal angles of tilt $\alpha$ and $\beta$ in the two directions (Figure 2), the Coulomb friction between liver and plates and the speed vit of the upper plate. Each of these parameters was changed separately, the others remaining at their optimal value. Values taken by the angles of tilt were 1,2 and 3 deg positively and negatively (6 runs), those taken by upper plate speeds were $0.001,0.005,0.01,0.05$, 0.1 and $0.5 \mathrm{~m} / \mathrm{s}$ and the Coulomb friction coefficient varied from 0.01 to 0.1 .

\subsubsection{Computing parameters}

Isolated parameters influencing the computing process, and then the model response, are the elements order and the calculation time step. For the elements order, number of integration points was changed from 1 to 4 . For the calculation time step, its definition is of great interest because it can also accelerate running process. Instead of keeping it free, eleven time steps are tested: the mean time step of the optimised model run $(0.04 \mathrm{~ms})$ and ten multiples of the maximum time step $(0.1,0.2,0.3,0.4$, 0.5, 0.6, 0.7, 0.8, 0.9 and $1 \mathrm{~ms})$ reached by the optimised model.

\section{Results}

\subsection{Inverse analysis}

\subsubsection{Influent parameters}

For the 16 treatments of the exploration step, the evaluation function took values from 0.555 to 13.6 . Results of the linear regression show that the model was independent of the density ( $\mathrm{p}$-value $=0.6)$. On the other hand, with the used behaviour law the model exhibited a high sensitivity to $v(\mathrm{p}$-value $=0.003), C_{10}(\mathrm{p}$-value $=0.0005)$ and $C_{01}(\mathrm{p}$ - 
value $=0.0002)$ with a high dispersion of the responses (Figure 3 ). The initial slope of curves increased with the modules and decreased with $v$. Finally, as $C_{10}$ and $C_{01}$ had a similar effect, their variation ranges were assumed as the same. Three configurations were then selected, giving the following variation ranges: $C_{10}$ and $C_{01}$ between 0.001 and $0.005 \mathrm{MPa}$ and $v$ between 0.2 and 0.495 .

\subsubsection{Optimal set of parameters}

Optimisation is performed on the three influent parameters $v, C_{10}$ and $C_{01}$ within the variation ranges given previously. Confrontation with the experimental curve is made only on the elastic phase of the compression process where damage has not occurred (Erreur ! Source du renvoi introuvable.) as failure and damage are not integrated in the model yet. After 25 runs of the NLPQL algorithm, the optimised set of parameters is: $v=0.4, C_{10}=0.005 \mathrm{MPa}$ and $C_{01}=0.003 \mathrm{MPa}$. For this set of parameters, the optimisation function scored 0.18 , which represents a suitable deviation between experiment and simulation in the elastic phase.

\subsection{Robustness analysis}

\subsubsection{Mechanical parameters influence}

With the three influent mechanical parameters, the space of study for robustness analysis is three-dimensional. According to the Optimal Latin Hypercube algorithm, 20 simulations are enough to describe efficiently the $10 \%$-variation space then defined. The mean value of the difference with optimised model computed is $5.1 \%$ with a minimum at $0.9 \%$ and a maximum at $10.8 \%$. Response curves keep the same shape as the optimal one and are well distributed around it without absurd response (Erreur ! Source du renvoi introuvable.). Hence, the optimised model can be considered as stable with regard to those parameters in the neighbourhood of their optimal values. 


\subsubsection{Boundary conditions influence}

The angle of tilt influence on the model response exhibits the liver asymmetry incidence. The response is less influenced by the angle of tilt for the positive values of $\alpha$ and $\beta$ than for the negative ones (Erreur ! Source du renvoi introuvable.). As it could be expected, the more the angle of tilt increases, the more the difference with the optimised model is important (tilt being positive or negative). It was observed that $\beta$-tilt has a much higher influence than $\alpha$-tilt.

The loading speed does not have any major incidence up to $0.1 \mathrm{~m} / \mathrm{s}$. With 0.5 $\mathrm{m} / \mathrm{s}$ the structure behaviour show oscillations around reference and lower speed curves (Erreur ! Source du renvoi introuvable.).

\subsubsection{Interactions description influence}

The differences between tests and optimised model observed for all the modifications made on the interface descriptions are range from 0.7 to $3.6 \%$. Those modelling parameters have thus no significant influence on optimized model response in the studied variations ranges.

\subsubsection{Computing parameters}

With mesh element formulation, the volume elements order does show a $1.88 \%$ difference between first and second order. Then, the optimised model meshed with 4 $\mathrm{mm}$ first order elements is robust in regard to the mesh.

Keeping the time step fixed or free is a so strong modelling hypothesis that the model cannot give results for a time step over $0.7 \mathrm{~ms}$. Below this value, difference between tests and optimised model responses is larger than $10 \%$ for time steps bigger than $0.3 \mathrm{~ms}$. Between 0.3 and $0.7 \mathrm{~ms}$, model response oscillates around optimised model response and is even jammed by noise. In conclusion, model robustness in regard to this parameter is limited and suggests keeping a free time step. 


\section{Discussion}

This work investigates human liver behaviour from experimental investigation under compression to behaviour law identification in a generic finite element model. The methodology focused on inverse identification method coupling exploration, optimisation and robustness analysis. Results lead to consider liver under quasi-static loadings as a hyperelastic structure described with a Mooney-Rivlin law.

\subsection{On the liver behaviour modelling}

The liver properties identification was performed on the whole liver organ. This choice was motivated by the willing to take into account the structure effects such as geometry or Glisson's capsule incidence. This study should be extended by the investigation of liver morphology incidence on its behaviour and the incidence of the hepatic vascular tree (Kerdok et al. 2006).

The parenchyma mechanical behaviour was described using a hyperelastic behaviour law with a Mooney-Rivlin model; capsule behaviour being set using specific complementary data (Brunon et al. 2010). With this law, the identification process was facilitated due to the limited number of parameters to optimize. According to the space of admissible parameters for optimisation and optimisation results, the obtained parameters set is relevant with physical properties of the liver described in the literature (Saraf et al. 2007). Additionally to describe high non linearity of loading, the Mooney-Rivlin law can be extended to the second order if needed. This liver model could be improved with behaviour law taking into account for the viscous effects of the structure (Miller 2000), and considering damage phenomenon up to the failure.

\subsection{On the inverse analysis approach}

The identification methodology used in this work was based on the coupling between exploration of solution space and optimisation on the relevant parameters. This two 
steps method allows focussing on the influent parameters and reducing of the admissible solution space before optimisation. So the optimisation step required a lower number of iterations and is focused on a dedicated part of the search space.

As most of optimisation technique, it remains the possibility to converge to a local optimum without any physical relevancy. The NLPQL optimisation algorithm used in this work is based on SQP method (Schittkowski 1985-1986). The choice of such a method leaves the opportunity to add optimisation constrains for further calibrations of the damageable model (maximal relative displacement or force at the first failure time for example). Nevertheless a gradient method could also be used for the optimisation method treated here. The 0.18 of the evaluation function for the optimal set of parameter allows a suitable description of the experimental response. This score could be improved both by using a more dedicated law (as describe previously), improving liver structure description and running a new time this optimisation process. The method presented here could also be improved by modifying the sampling method used for robustness analysis considering robust design methods (Zang et al. 2005).

\subsection{On the model robustness}

The robustness analysis on model mechanical parameters shows a good stability with a mean dispersion of $5.1 \%$. This point ensures a good reliability of the model response. The robustness of the model response is also dependent on the testing conditions. The angle of tilt incidence on model response seems to be strongly dependent to the intrinsic geometry of the liver and its positioning during solicitations. The model response could be considered as stable with an angle of tilt ranged between -2 to $2^{\circ}$. This condition should be also carefully respected during experiments to keep results consistency. With loading speed, the low sensitivity of the 
model up to $0.1 \mathrm{~m} / \mathrm{s}$ of imposed velocity let to increase numerical loading speed to improve computation time.

The time step control during calculation could induce some discrepancy in model results. This point is strongly related to the explicit FE algorithm used with Radioss software. This analysis suggests keeping a free time step during the optimisation process whatever the cost for calculation time.

The robustness analysis performed could further integrate the coupled effects of the studied modelling parameters. For example, it has been showed that the mesh size influence on a FE model response could depend on loading speed variations (Schmidt et al. 2009).

At least, the variability induced by modelling parameters is quantified within a reasonable time: calculus time necessary for the whole exploration, optimisation and robustness analysis process does not exceed a week using six processors in parallel.

\section{Conclusion}

This study shows the feasibility of an integrated approach coupling exploration, optimisation and robustness analysis for a FE model of the liver in compression with a hyperelastic parenchyma. The model successfully fits experimental data and is robust enough to take into account the experimental variability. This methodology will thus be extended to more complex model such as hyper-viscoelastic model including damage and failure so as to characterize liver, or other human organs behaviour under impact situations.

\section{References}

Brunon A, Bruyère-Garnier K and Coret M. 2010. Mechanical characterization of liver capsule through uniaxial quasi-static tensile tests until failure. J Biomech. 43(11):2221-2227. 
Carter FJ, Frank TG, Davies PJ, McLean D and Cuschieri A. 2001. Measurements and modelling of the compliance of human and porcine organs. Med Image Anal. 5(4):231-236.

Chui C, Kobayashi E, Chen X, Hisada T and Sakuma I. 2007. Transversely isotropic properties of porcine liver tissue: experiments and constitutive modelling. Med Biol Eng Comput. 45:99-106.

Durand-Reville M, Tillier Y, Paccini A, Lefloch A, Delotte J, Bongain A and Chenot JL. 2004. Immediate post-operative procedure for identification of the rheological parameters of biological soft tissue. Int Congr Ser. 1268:407-412.

Egorov V, Tsyuryupa S, Kanilo S, Kogit M and Sarvazyan A. 2008. Soft tissue elastometer. Med Eng Phys. 30(2):206-212.

Farshad M, Barbezat M, Flüeler P, Schmidlin F, Graber P and Niederer P. 1999. Material characterization of the pig kidney in relation with the biomechanical analysis of renal trauma. J Biomech. 32(4):417-425.

Harrison SM, Bush MB and Petros PE. 2007. A pinch elastometer for soft tissue. Med Eng Phys. 29(3):307-315.

Haug E, Choi H-Y, Robin S, Beaugonin M and Ayache N. 2004. Human Models for Crash and Impact Simulation. Handbook of Numerical Analysis. 12:231-452.

Kerdok AE, Ottensmeyer MP and Howe RD. 2006. Effects of perfusion on the viscoelastic characteristics of liver. J Biomech. 39(12):2221-2231.

Labé A, Arnoux P-J, Behr M, Kayvantash K and Brunet C. 2006. Advanced finite element model to simulate pelvic failure process. Paper presented at: 7 th international symposium on Computer Methods in Biomechanics; Nice, France.

Laumon B. 2002. Recherches coordonnées sur les traumatismes consécutifs à un accident de la circulation routière, leurs causes et conséquences. Rapport UMRETTE $n^{\circ}$ 0205: INRETS/Université Claude Bernard Lyon 1 - Predit 1996-2000.

Mazza E, Nava A, Hahnloser D, Jochum W and Bajka M. 2007. The mechanical response of human liver and its relation to histology: An in vivo study. Med Image Anal. 11(6):663-672.

Miller K and Chinzei K. 1997. Constitutive modelling of brain tissue: Experiment and theory. J Biomech. 30(11-12):1115-1121.

Miller K. 2000. Constitutive modelling of abdominal organs. J Biomech. 33(3):367373.

Miller K. 2005. Method of testing very soft biological tissues in compression. J Biomech. 38(1):153-158.

Netter FH. Atlas d'anatomie humaine. 2nd edition. Norvatis.

Park J-S. 1994. Optimal Latin-hypercube designs for computer experiments. J Stat Plan Infer. 39(1):95-111.

Sacks J, Welch WJ, Mitchell TJ and Wynn HP. 1989. Design and analysis of computer experiments. Stat Sci. 4(4):409-423.

Saraf H, Ramesh KT, Lennon AM, Merkle AC and Roberts JC. 2007. Mechanical properties of soft human tissues under dynamic loading. J Biomech. 40(9):1960-1967.

Schittkowski K. 1985-1986. NLPQL: A Fortran subroutine for solving constrained nonlinear programming problems. Ann Oper Res. 5:485-500.

Schmidt H, Alber T, Wehner T, Blakytny R and Wilke H-J. 2009. Discretization error when using finite element models: Analysis and evaluation of an underestimated problem. J Biomech. 42(12):1926-1934. 
Schmidt SR and Launsby RG. 1994. Understanding industrial designed experiments. 4th edition. Colorado Spring: Air Academy Press.

Snedeker JG, Barbezat M, Niederer P, Schmidlin FR and Farshad M. 2005. Strain energy density as a rupture criterion for the kidney: impact tests on porcine organs, finite element simulation, and a baseline comparison between human and porcine tissues. J Biomech. 38(5):993-1001.

Tillier Y, Paccini A, Durand-Reville M, Bay F and Chenot JL. 2003. Threedimensional finite element modelling for soft tissues surgery. Int Congr Ser. 1256:349-355.

Winckler G. 1974. Manuel d'Anatomie Topographique et Fonctionnelle. 2nd edition. Paris: Masson.

Zang C, Friswell MI and Mottershead JE. 2005. A review of robust optimal design and its application in dynamics. Comput Struct. 83(4-5):315-326. 

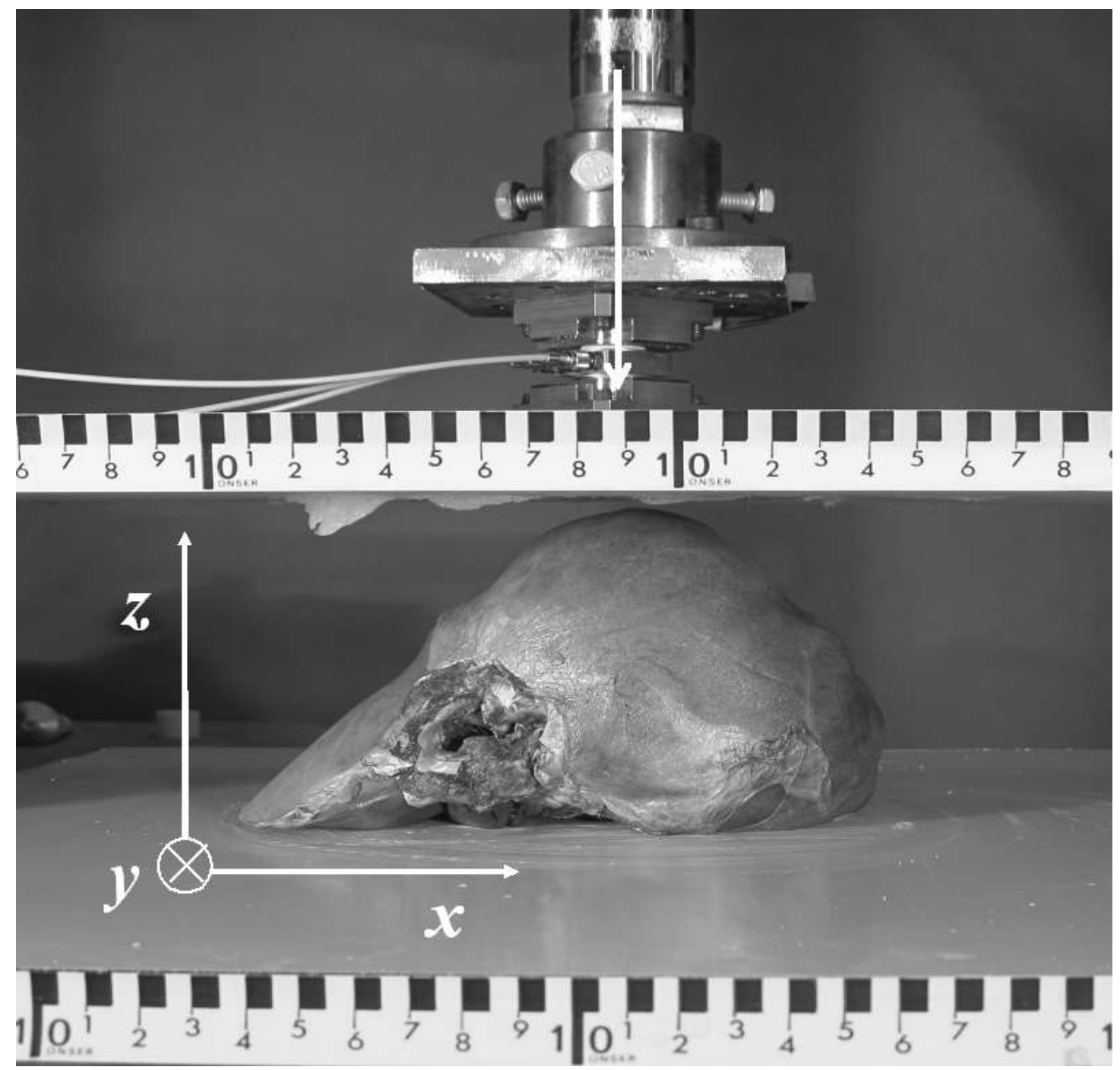

Figure 1. Experimental set up for the uniaxial liver compression. The upper plate goes down at a constant velocity toward the anterior face of the liver. 

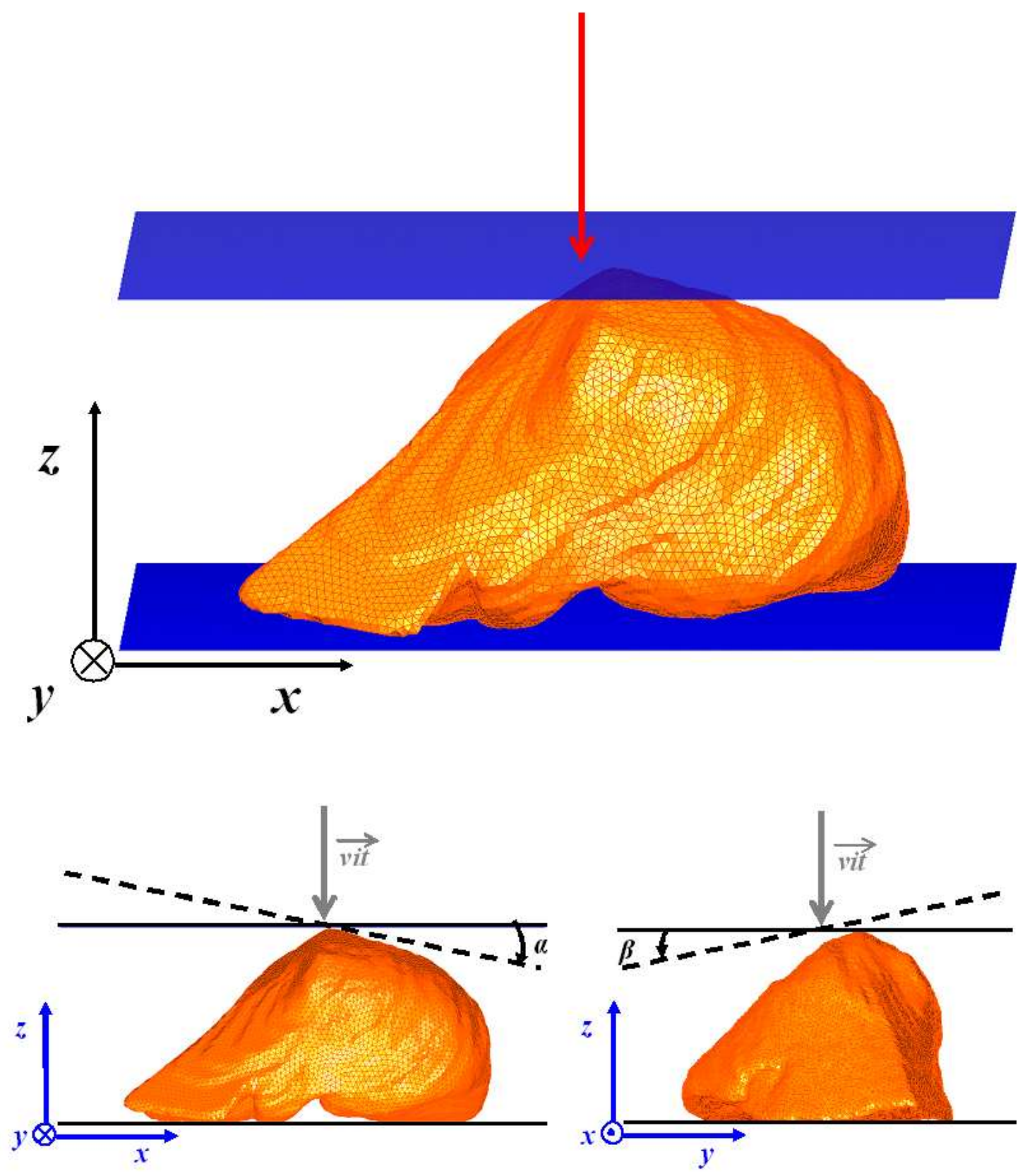

Figure 2. Upper plate speed and angles of tilt. a) Front view of the initial model with $\alpha$ $=0$ and $\beta=0$. b) Definition of $\alpha$ and $\beta$ 


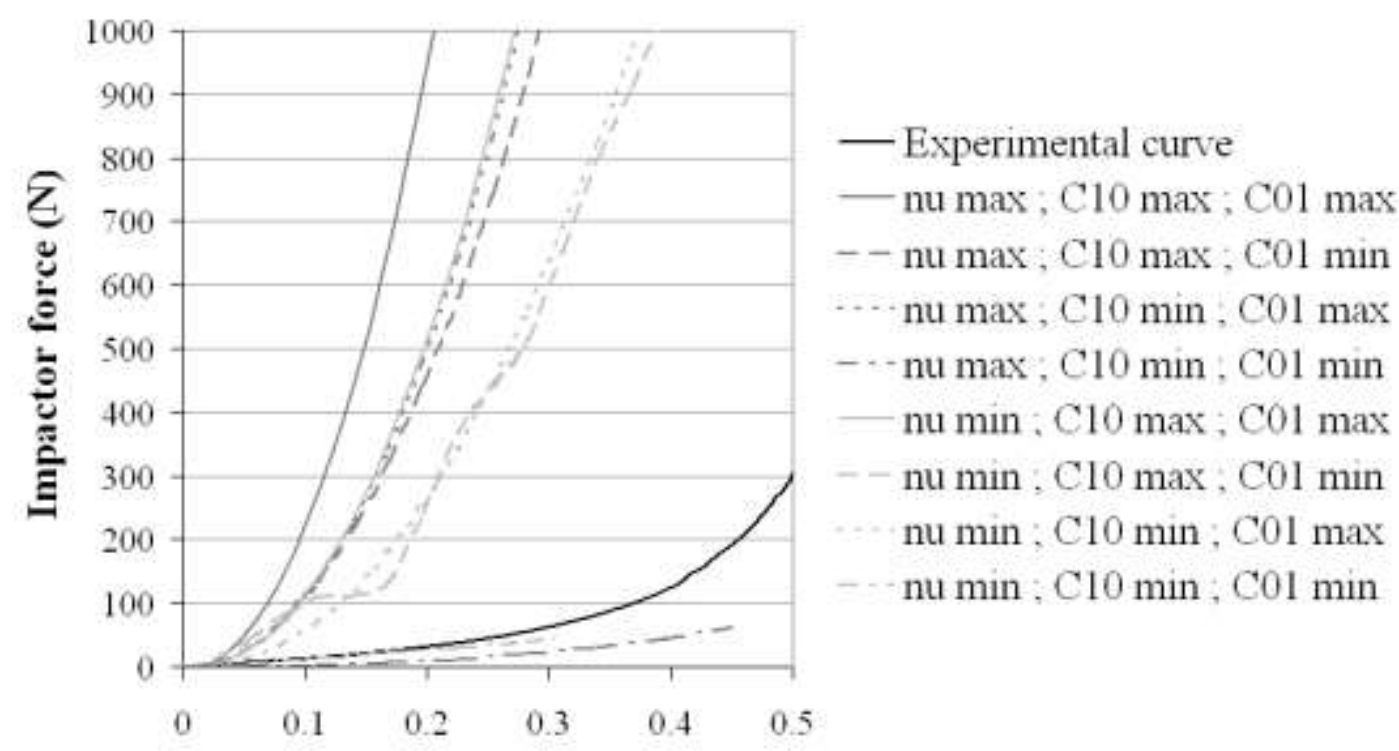

Impactor relative displacement

Figure 2. Responses of the influent treatments of the exploration step.

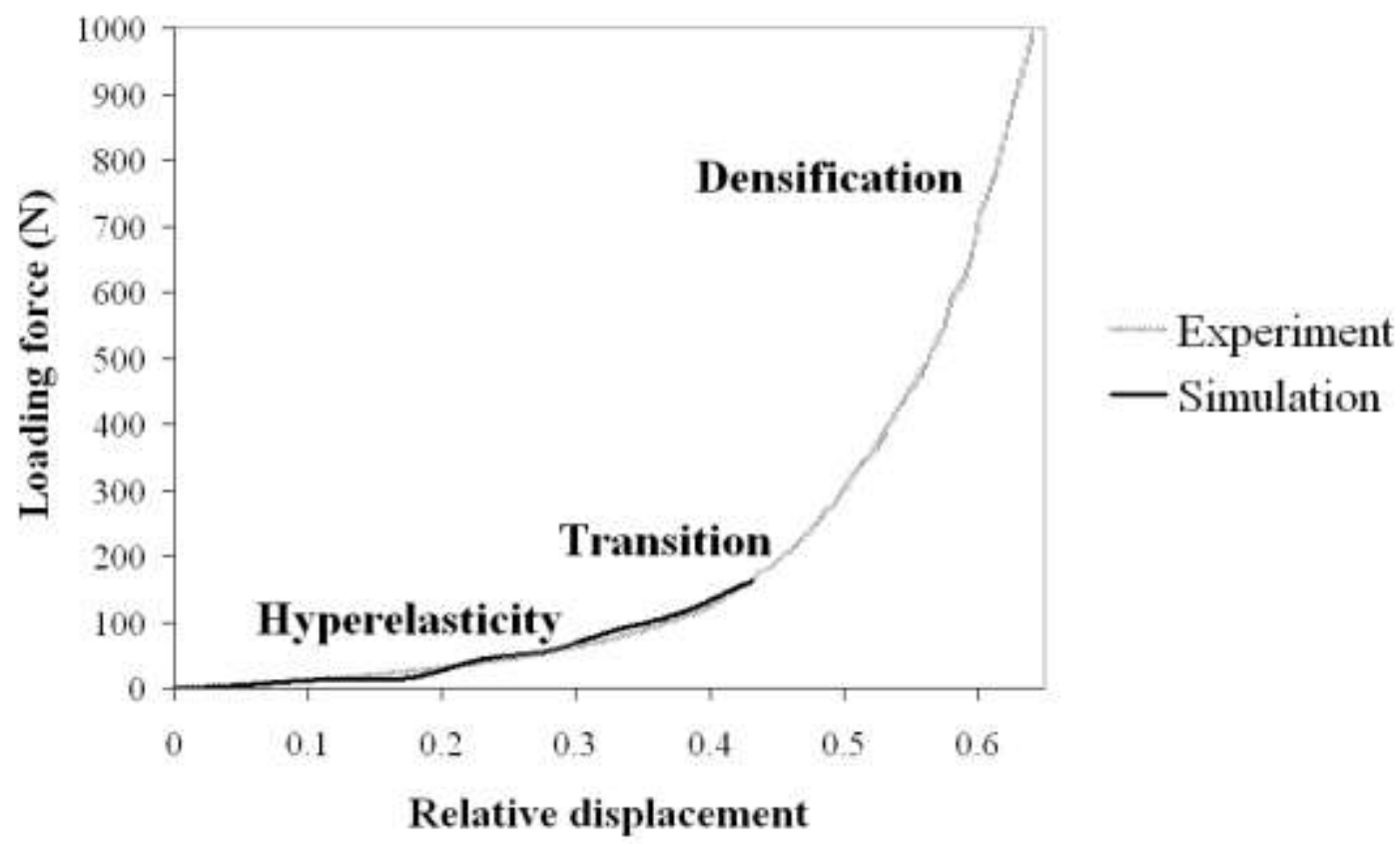

Figure 3. Response of the optimised model in the elastic phase of the compression. 


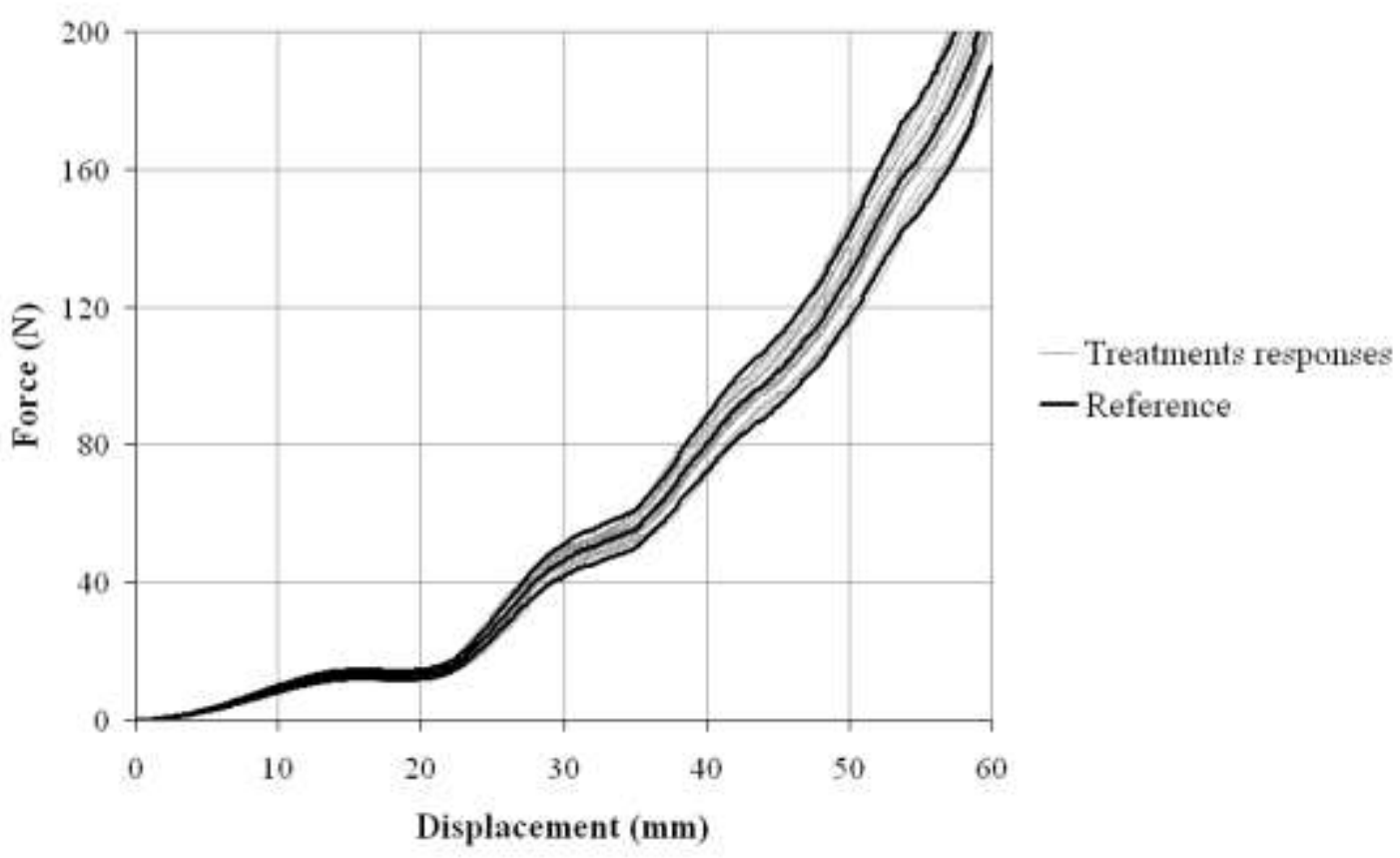

Figure 4. Responses of the different simulations of analysis of model robustness on mechanical parameters. The reference corridor is described by three curves: the one from the optimised model (at the middle) and the two obtained by adding or deducting $10 \%$ of the first one.

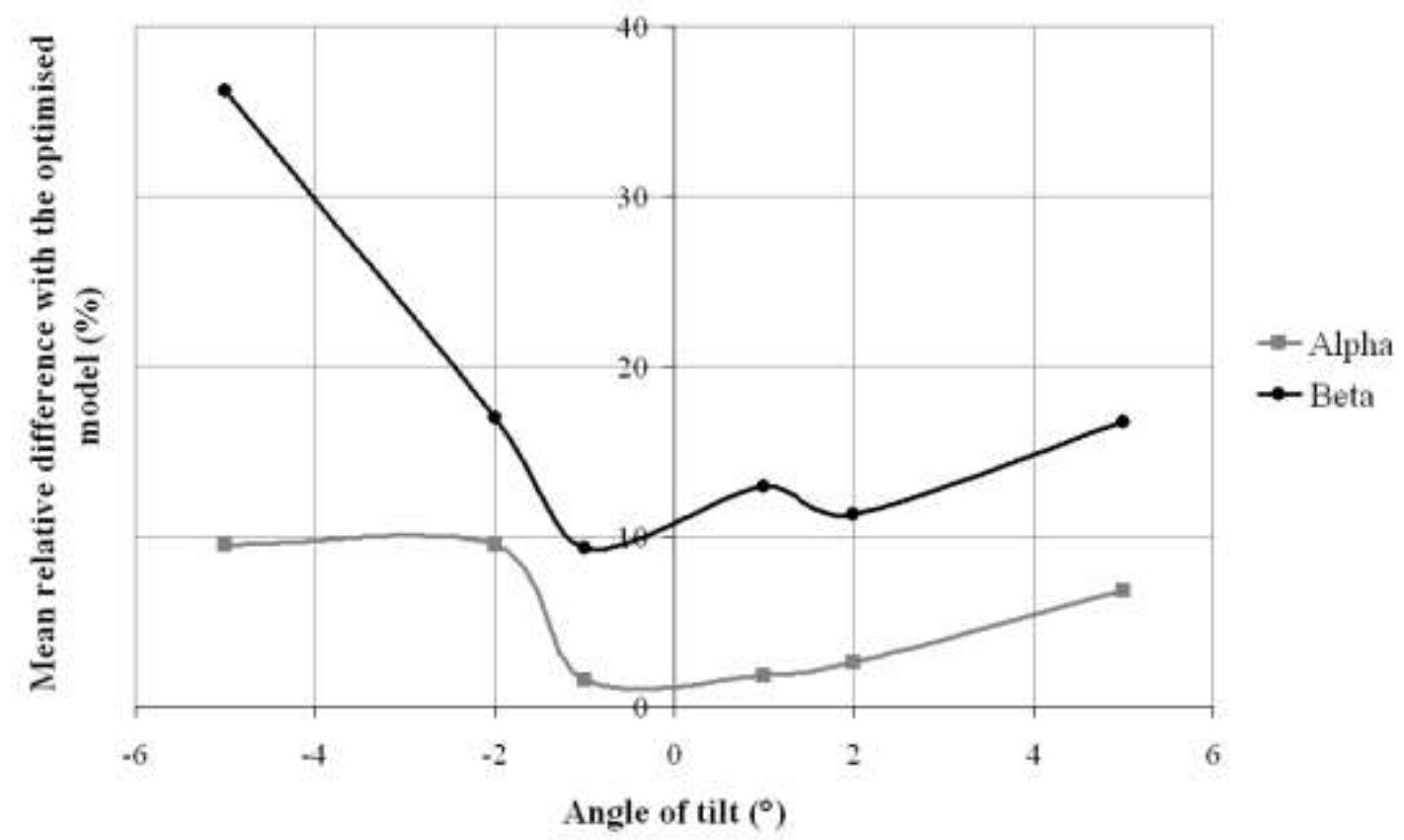

Figure 5. Influence of the upper plate angle of tilt on the difference with the optimised model. 


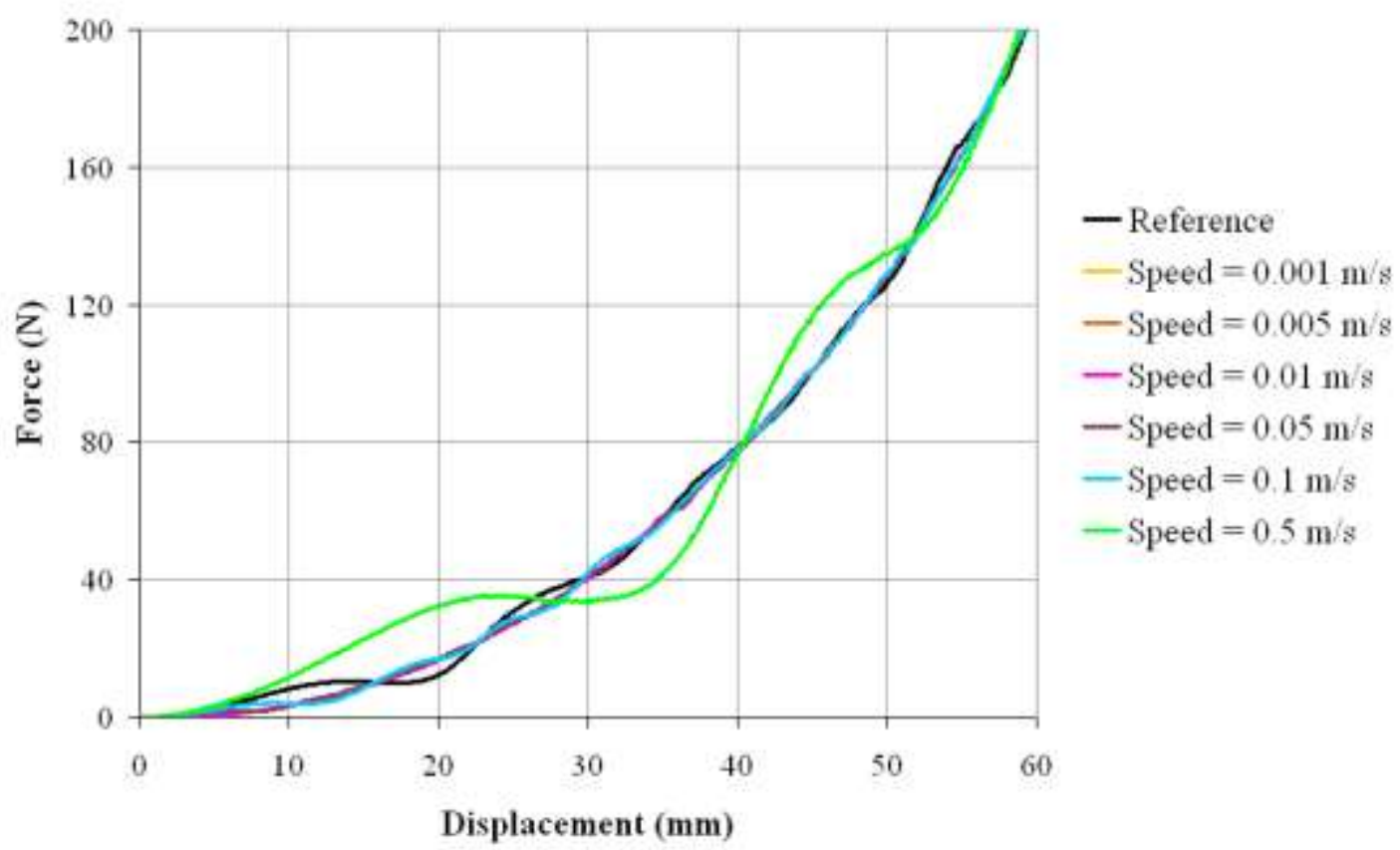

Figure 6. Responses of simulations at different solicitation speeds. 